\title{
Influencia de la maduración y el tipo de cocinado en la valoración sensorial y el perfil de compuestos aromáticos del cruce industrial de raza Retinta
}

\author{
B. Panea ${ }^{1, *}$, G. Ripoll ${ }^{1}$, K. Insausti², M.J. Beriain ${ }^{2}$, C. Sañudo ${ }^{3}$ y P. Albertí ${ }^{1}$ \\ 1 Centro de Investigación y Tecnología Agroalimentaria de Aragón, Instituto Agroalimentario de Ara- \\ gón - IA2 (CITA-Universidad de Zaragoza). Avenida de Montañana, 930, 50059, Zaragoza, España \\ 2 Escuela Técnica Superior de Ingenieros Agrónomos, Universidad Pública de Navarra. 31006 Pamplo- \\ na, España \\ 3 Facultad de Veterinaria, Universidad de Zaragoza, Instituto Agroalimentario de Aragón - IA2 (CITA- \\ Universidad de Zaragoza). C/ Miguel Servet, 176, 50013, Zaragoza, España
}

\section{Resumen}

Se estudió el efecto del tiempo de maduración y tipo de cocinado sobre la valoración sensorial y el perfil de los compuestos aromáticos de la carne de animales de los cruces de toros de las razas Retinta, Limousine, Pirenaica y Asturiana de los Valles con vacas Retintas. Se utilizaron dos paneles, uno para el efecto de la maduración y otro para el tipo de cocinado. El entrenamiento de los catadores y la temperatura de cocinado fueron idénticos en ambos casos, aunque los perfiles difieren entre sí. En la valoración sensorial no se encontraron interacciones entre efectos. El cruce sólo afectó a la terneza, siendo la carne de Pirenaica x Retinta y Retinta x Retinta algo más dura. La terneza, el flavor a hígado y el flavor ácido aumentaron con la maduración, al contrario que la jugosidad. El tipo de cocinado afectó a la terneza y a la facilidad al tragar, estando ambas variables inversamente correlacionadas. Se identificaron 50 compuestos aromáticos. El cruce tuvo menos influencia que el tipo de cocinado y se encontró una interacción significativa entre efectos para 15 de los compuestos, especialmente hidrocarburos. El cruce afectó al porcentaje de alcoholes, compuestos azufrados y cetonas pero no al resto y el tipo de cocinado, a todos los compuestos. Los hidrocarburos y las cetonas presentaron mayores concentraciones que el resto. Para todos los grupos de compuestos excepto para los azufrados, las cantidades detectadas fueron mayores cuando el método de cocinado fue la cocción.

Palabras clave: Panel, compuestos aromáticos, vacuno.

Influence of ageing and cooking method on sensory evaluation and aromatic compounds profile of meat from Retinta breed crossbreeding

\begin{abstract}
We studied the effect of ageing period and cooking method on sensory evaluation and aromatic compounds profile of meat from crosses of Retinta, Limousine, Pirenaica and Asturiana de los Valles bulls and Retinta dams. Two panels were used, one to study the ageing effect and another to compare the effect of cooking method. The training and temperature of cooking were identical in both cases, although the descriptive profiles are different among themselves. No interaction between effects was found in sensory evaluation. Crossbreeding only affected tenderness, being meat from Pirenaica $x$ Re-
\end{abstract}

\footnotetext{
* Autor para correspondencia: bpanea@aragon.es

Cita del artículo: Panea B, Ripoll G, Insausti K, Beriain MJ, Sañudo C, Albertí P (2019). Influencia de la maduración y el tipo de cocinado en la valoración sensorial y el perfil de compuestos aromáticos del cruce industrial de raza Retinta. ITEA-Información Técnica Económica Agraria 115(2): 149-162. https://doi.org/10.12706/itea.2018.030
} 
tinta and Retinta $x$ Retinta crosses slightly tougher. Tenderness, liver flavour and acid flavour increased with ageing, contrary to juiciness. The cooking method affected tenderness and ease swallowing, and both variables were inversely correlated. 50 aromatics compounds were identified and we found a significant interaction effects for 15 compounds, especially hydrocarbons. Crossbreeding affected the percentage of alcohols, ketones and sulphur compounds whereas cooking method affected to all compounds. Hydrocarbons and ketones showed higher concentrations than the rest of the compounds. All compounds except the ketone groups were affected by cooking method. For all groups of compounds except for the sulphur, the amounts detected were higher when the cooking method was water-boiling.

Keywords: Beef, panel test, aromatic compounds.

\section{Introducción}

La adhesión de España a la UE facilitó la introducción en España de razas extranjeras con unas características carniceras superiores a las de las razas autóctonas y originó que el cruzamiento industrial fuera práctica común (Ahumada, 1997). En la actualidad, aproximadamente el $35 \%$ del ganado sacrificado en España está cruzado en cierto grado siendo el cruce más frecuente el de hembras autóctonas con machos especializados de aptitud cárnica (Illescas et al., 2009). El impacto de la heterosis en los parámetros productivos es conocido desde hace mucho tiempo y se han desarrollado programas de cruzamientos en todas las especies ganaderas para optimizar su beneficio (Dickerson, 1973). El macho de raza seleccionada aporta canales con mayor conformación y más rendimiento carnicero, mientras que la nodriza de raza rústica o ambiental asegura un aprovechamiento de los recursos locales por su adaptación al medio. Sin embargo, el efecto del cruzamiento sobre la calidad de la carne no está suficientemente estudiado, a pesar de que la base genética condiciona la cantidad y tipo de fibras musculares, la cantidad de mioglobina del músculo, el grado de engrasamiento de la carne y el perfil de ácidos grasos (Wood et al., 2008; Gama et al., 2013; Font i Furnols y Guerrero, 2014; Khan et al., 2015). Todos estos factores tienen repercusión sobre los procesos de oxidación que ocurren durante el al- macenamiento de la carne. Los procesos de oxidación proteica y lipídica originan numerosos compuestos responsables del sabor y flavor de la carne (Mottram, 1998) y se modifican con la maduración y el cocinado (Campo et al., 1999; Wood et al., 2004; Ripoll et al., 2014). Así, la ruptura de las miofibrillas durante la maduración da lugar a aminoácidos y péptidos (Ngapo et al., 2013) que en cocinados rápidos a alta temperatura forman los derivados de la reacción de Maillard, mientras que los cocinados lentos y a bajas temperaturas dan lugar a compuestos aromáticos derivados de los lípidos (Kerth y Miller, 2015).

El objetivo del presente estudio fue estudiar la influencia de la maduración y el tipo de cocinado sobre la valoración sensorial y el perfil de compuestos aromáticos de la carne de terneros procedentes del cruce industrial de vacas de raza Retinta.

\section{Material y métodos}

El presente trabajo se deriva del proyecto RTA 2001-106. Los resultados del análisis instrumental de la carne de los animales utilizados han sido publicados en Panea et al. (2016), y muestran que no existe efecto del cruce ni en la cantidad de grasa intramuscular ni el porcentaje de proteína de la carne de estos animales. 


\section{Manejo de los animales}

Todos los procedimientos realizados han sido aprobados por el Comité Ético de Experimentación Animal del Centro de Investigación y Tecnología Agroalimentaria de Aragón (CITA). El cuidado y el uso de los animales han sido desarrollados de acuerdo con la Directiva de la Unión Europea n²010/63 de la protección de animales utilizados en experimentos y otros propósitos científicos (E.U., 2010).

Se cebaron 28 terneros, repartidos en cuatro lotes: 7 de Retinta en pureza (RExRE), 8 del cruce Limousine $x$ Retinta (LIxRE), 6 del cruce Pirenaica x Retinta (PIxRE) y 7 del cruce Asturiana de los Valles $x$ Retinta (ASxRE). A su llegada a las instalaciones del CITA en Zaragoza, los animales fueron desparasitados y vacunados contra enfermedades respiratorias y toxemias. Tras un periodo de adaptación, a los animales se les ofreció pienso concentrado (Tabla 1) y paja ad libitum. Los animales se sacrificaron a los doce meses de edad (362 \pm 11,0 días) con $480,0 \pm 15,5 \mathrm{~kg}$ de peso vivo de media. El sacrificio se realizó en un matadero oficial (Mercazaragoza, Zaragoza, España) a $6 \mathrm{~km}$ de las instalaciones del CITA. Para minimizar el estrés anterior al sacrificio los animales se transportaron el mismo día del sacrificio y se sacrificaron inmediatamente a su llegada, sin ayuno, previo aturdimiento con una pistola de émbolo cautivo. Una vez faenados los animales, las canales se mantuvieron en refrigeración en cámara a $4^{\circ} \mathrm{C}$ durante 24 horas. Posteriormente, se extrajo de la media canal izquierda la porción de músculo Longissimus thoracis et lumborum comprendido entre la $10^{a}$ vértebra torácica y la $6^{\text {a }}$ lumbar y se obtuvieron 12 filetes de 2 $\mathrm{cm}$ de espesor. El proyecto del que deriva este trabajo es un proyecto coordinado, por lo que el análisis sensorial se realizó en dos laboratorios diferentes: en la Universidad de Zaragoza se realizó una prueba para estudiar el efecto del tiempo de maduración mientras
Tabla 1. Composición del pienso utilizado en el cebo de animales procedentes de distintos cruces de Retinta.

Table 1. Feed composition used to raise animals from different Retinta crosses.

\begin{tabular}{lc}
\hline Ingredientes & Porcentaje \\
\hline Maíz & 35,0 \\
Cebada 10\% & 16,11 \\
Soja 44\% & 9,48 \\
Tercerillas & 6,0 \\
Gluten feed & 12,0 \\
Cascarilla soja & 8,0 \\
Germen maíz & 5,0 \\
Asigras (buffer) & 0,46 \\
Pulpa remolacha & 3,0 \\
Carbonato cálcico & 1,38 \\
Aceite de palma & 2,0 \\
Fosfato bicálcico & 0,45 \\
Sal & 0,6 \\
Corrector vitamínico & 0,5 \\
Materia seca (\% MS) & 88,21 \\
Grasa bruta (\% MS) & 6,02 \\
Proteína bruta (\% MS) & 13,75 \\
Energía (MJ EM/ kg MS) & 12,55 \\
\hline
\end{tabular}

que en la Universidad Pública de Navarra la prueba se diseñó para comparar el efecto del tipo de cocinado. Por lo tanto, los filetes se maduraron al vacío 2, 7 ó 21 días, en función del diseño experimental de cada laboratorio. Tras la maduración, todas las muestras se congelaron a $-18^{\circ} \mathrm{C}$, manteniéndose así hasta el día del análisis y se descongelaron a $4^{\circ} \mathrm{C}$ durante $24 \mathrm{~h}$. En cada laboratorio se realizó una sesión previa para elaborar el perfil descriptivo, por lo que éstos son distintos 
en función del laboratorio, pero el entrenamiento de los catadores (ISO-8586:2012) y las temperaturas de cocinado $\left(70^{\circ} \mathrm{C}\right.$ de temperatura interna) fueron idénticos en ambos casos.

\section{Efecto de la maduración}

Esta prueba se realizó en la sala de catas de la facultad de Veterinaria de Zaragoza. Se utilizaron tres filetes por animal, madurados a 1, 7 ó 21 días. Una vez descongelados, los filetes se envolvieron en papel de aluminio y se cocinaron en un grill de doble placa (SAMMIC P8D-2) precalentado a $200^{\circ} \mathrm{C}$ hasta alcanzar una temperatura interna de $70^{\circ} \mathrm{C}$, controlada con un termopar (Jenway 2000, Staffordshire, Reino Unido). Una vez cocinados, se retiró la grasa y el tejido conectivo externo, se cortaron en muestras cúbicas de 2 $\mathrm{cm}$ de lado, se envolvieron en papel de aluminio con un código de tres dígitos y se mantuvieron a $60^{\circ} \mathrm{C}$ un máximo de cinco minutos hasta que se sirvieron. Las muestras se sirvieron a un panel entrenado de ocho miembros en cabinas individuales con luz roja para enmascarar diferencias en el color de la carne (ISO-8586:2012). Para evitar efectos del orden de presentación, las muestras se presentaron en distinto orden a cada catador (MacFie et al., 1989). Para limpiar el paladar, cada catador tenía a su disposición agua y pan. Se realizó un test comparativo multimuestra, con tres/cuatro muestras por plato, por medio de un diseño de bloque equilibrado incompleto (Meilgaard et al., 1991). Se realizaron 14 sesiones con un total de 162 evaluaciones por catador. Los descriptores utilizados fueron intensidad de olor a vacuno, intensidad de olor a hígado, jugosidad inicial, cantidad de residuo, intensidad de flavor a vacuno, intensidad de flavor a hígado e intensidad de flavor ácido. Cada uno de los atributos se valoró utilizando una escala lineal no estructurada en la que 1 correspondía al valor más bajo y 10 al más alto para el atributo en cuestión.

\section{Efecto del tipo de cocinado}

Esta prueba se realizó en el laboratorio de la Universidad Pública de Navarra. Todos los filetes utilizados tenían una maduración de 7 días. Las muestras se cocinaron por tres métodos diferentes: cocción en baño de agua (Selecta Precistern, Barcelona, España), plancha de doble placa (Silanos, Barcelona, España) precalentada a $200^{\circ} \mathrm{C}$ y asado en horno de convección (BSH HM21211 DUO 413, Zaragoza, España). Todas las muestras se cocinaron hasta que alcanzaron una temperatura interna de $70^{\circ} \mathrm{C}$ (AMSA, 2016). La temperatura interna se monitorizó con la ayuda de un termopar (Digitron, Hertford, UK). Las muestras se evaluaron con un panel entrenado de 11 miembros entrenados según la ISO-8586:2012. Los panelistas valoraron jugosidad inicial y continuada, dureza, cohesividad, facilidad para tragar, untuosidad, intensidad global de aroma, intensidad de aroma a hígado, intensidad de flavor a hígado, intensidad de flavor a sangre, intensidad de flavor a grasa e intensidad de flavor residual. Se utilizó una escala lineal no estructurada de 10 puntos en la que 1 correspondía al valor más bajo y 10 al más alto para el atributo en cuestión.

\section{Compuestos volátiles}

El análisis de compuestos volátiles se realizó sobre las muestras utilizadas para el panel de cata de la Universidad Pública de Navarra. Se estudiaron tanto en carne cruda como en carne cocinada mediante cromatografía de gases y espacio de cabeza dinámico (Gorraiz et al., 2002). Aproximadamente $10 \mathrm{~g}$ de muestra picada se colocaron en un vial con espacio de cabeza. El vial fue conectado a un concentrador de purga y trampa (purge-and-trap, 4460A, OI Analytical, College Station, Texas). La muestra fue calentada a $70^{\circ} \mathrm{C}$ y purgada con $40 \mathrm{~mL} / \mathrm{min}$ de flujo de helio durante 10 minutos. Los volátiles del espacio de cabeza se recogieron en una trampa Tenax GC (60/80 
mesh) a $30^{\circ} \mathrm{C}$ y sufrieron una desorción térmica a $180^{\circ} \mathrm{C} / 4$ minutos con $40 \mathrm{~mL} / \mathrm{min}$ de flujo de helio. Se utilizó un cromatógrafo de gases HP-5890 (Hewlett-Packard, Madrid, España) equipado con una columna capilar HP5. El análisis se realizó utilizando helio como gas de arrastre. La columna se calentó inicialmente a $35^{\circ} \mathrm{C}$ durante 15 minutos y se programó para alcanzar $220^{\circ} \mathrm{C}$ con una rampa de $8^{\circ} \mathrm{C} /$ minuto, manteniéndose a esa temperatura durante 5 minutos. La determinación se hizo por duplicado para cada muestra. La identificación de los compuestos volátiles se hizo con un cromatógrafo de gases HP-6890 (Hewlett-Packard, Madrid, España) acoplado a un espectrómetro de masas cuádruple HP5973 (Hewlett-Packard, Madrid, España). Los parámetros operacionales más importantes del cromatógrafo de gases fueron los siguientes: voltaje inicial $70 \mathrm{eV}$; temperatura de la fuente de iones, $230^{\circ} \mathrm{C}$; voltaje del multiplicador de electrones, $3000 \mathrm{~V}$; velocidad de escaneado, 3,32 scan/s y rango de escaneado 30 a 250 uma, con el cuadrupolo a $108^{\circ} \mathrm{C}$. El espectro obtenido se comparó con la biblioteca Wiley 275. Los índices de retención se calcularon con el método Van Den Dool y Kratz (1963), modificado para cromatógrafos de gases programados por temperatura.

\section{Análisis estadístico}

El análisis estadístico se realizó utilizando el paquete estadístico SAS (versión 9.3). Se realizaron dos GLM, uno con el genotipo y tiempo de maduración como efectos fijos y otro con el genotipo y tipo de cocinado como efectos fijos. Para todas las variables se calcularon las medias y la comparación entre medias se hizo con el test de Duncan, con $p<0,05$.

\section{Resultados y discusión}

\section{Efecto del tiempo de maduración sobre la valoración sensorial}

En la Tabla 2 se muestran las medias y el error estándar de las variables medidas, así como el efecto del tiempo de maduración. La variabilidad de los datos del análisis sensorial del presente trabajo estaría de acuerdo con los datos ofrecidos por Campo et al. (1997), quienes señalan coeficientes de variación de entre el 8 y el $35 \%$. No se encontró efecto del cruce sobre ninguno de los atributos estudiados $(p>0,05)$ pero la maduración afectó a algunas de las variables. No se encontraron interacciones significativas entre efectos. Puede verse que la terneza aumentó $(p=0,000)$ con el tiempo de maduración, pero sólo durante la primera semana, para estabilizarse a partir del día 7. Por el contrario, la jugosidad disminuyó a partir del $7^{\circ}$ día $(p=0,000)$. El flavor a hígado $(p=0,007)$ y el flavor ácido aumentaron $(p=0,003)$ paulatinamente a lo largo de la maduración.

El efecto de la raza sobre la valoración sensorial de la carne está ampliamente estudiado, aunque los resultados pueden ser contradictorios. Así, algunos estudios muestran diferencias entre razas (Santolaria et al., 1997; Campo et al., 1999), mientras que otros no las encuentran (Albertí et al., 1995; Insausti et al., 2005). Vieira et al. (2006) señalan que la carne de los animales procedentes de cruce industrial con razas cárnicas es más tierna que la de los animales de tipo rústico criados en pureza, pero en el presente trabajo la terneza de la carne de la raza en pureza (RExRE) no fue significativamente diferente del resto. Sinclair et al. (1998), utilizando cruces de Angus o de Charolais como padres, demostraron que si todos los animales reciben la misma dieta, la raza no influye sobre las características organolépticas de la carne. 
Tabla 2. Medias, error estándar y significación del efecto maduración (valor de p) sobre la valoración sensorial de la carne de animales procedentes de distintos cruces de Retinta.

Table 2. Means, standard error and signification of the ageing effect ( $p$ values) on the sensory analysis of meat from different Retinta breed crosses.

\begin{tabular}{|c|c|c|c|c|c|}
\hline \multirow{2}{*}{ Variable* } & \multicolumn{3}{|c|}{ Maduración } & \multirow{2}{*}{ e.e. } & \multirow{2}{*}{ valor de $p$} \\
\hline & 1 día & 7 días & 21 días & & \\
\hline Olor vacuno & 5,33 & 5,38 & 5,30 & 0,070 & 0,757 \\
\hline Olor a hígado & 2,80 & 2,97 & 3,09 & 0,087 & 0,058 \\
\hline Terneza & $5,22^{b}$ & $6,11^{\mathrm{a}}$ & $6,22^{\mathrm{a}}$ & 0,072 & 0,000 \\
\hline Jugosidad Inicial & $4,85^{a}$ & $5,04^{a}$ & $4,42^{b}$ & 0,083 & 0,000 \\
\hline Residuo & 4,52 & 4,21 & 4,47 & 0,006 & 0,058 \\
\hline Flavor vacuno & 5,93 & 5,97 & 5,88 & 0,061 & 0,543 \\
\hline Flavor a hígado & $3,03^{b}$ & $3,23^{\mathrm{ab}}$ & $3,44^{\mathrm{a}}$ & 0,093 & 0,007 \\
\hline Flavor ácido & $3,35^{b}$ & $3,41^{b}$ & $3,74^{a}$ & 0,090 & 0,003 \\
\hline
\end{tabular}

* Escala de 1 a 10, siendo 1 el valor más bajo y 10 el más alto para la intensidad del atributo en cuestión. e.e.- error estándar. a, b.- superíndices distintos en la misma fila implican diferencias significativas entre tiempos de maduración $(p<0,05)$.

En cuanto a la maduración, está demostrado que tiene un marcado efecto sobre la calidad sensorial de la carne (Hughes et al., 2014) y que este efecto es más importante que el de otros factores como el genotipo o el nivel de engrasamiento (Albertí et al., 1995). La maduración aumenta la intensidad del flavor meálico, flavor ácido y flavor a rancio (Yancey et al., 2005), así como su terneza y jugosidad (Campo et al., 1999). Se ha descrito que la maduración origina un aumento de la intensidad del olor debida principalmente a la aparición de hidrocarburos alifáticos durante la oxidación de los lípidos, así como por el incremento de ácidos grasos libres producidos por lipolisis (Gorraiz et al., 2002). Insausti et al. (2002) describen que el flavor a hígado y flavor ácido se deben principalmente a las cetonas y algunos hidrocarburos como los octenos y octanos, compuestos que han sido detectados en el presente trabajo.

\section{Efecto del tipo de cocinado sobre la valoración sensorial}

En la Tabla 3 se presenta la significación del cruce y el tipo de cocinado sobre la valoración sensorial de la carne. El cruce sólo tuvo efecto sobre la terneza $(p=0,014)$, mientras que el cocinado afectó a la terneza $(p=$ $0,015)$ y a la facilidad al tragar $(p=0,001)$. No se encontraron interacciones entre efectos. La carne de los cruces PIxRE $(7,44)$ y RExRE $(7,12)$ fue algo más dura que la de los cruces LIxRE $(6,25)$ y ASxRE $(5,96)$ (Tabla 4). En cuanto al tipo de cocinado, puede verse en la tabla 5 que dureza y facilidad para tragar están inversamente relacionadas, coincidiendo con los resultados de otros autores (Campo et al., 1999; Panea, 2002; Panea et al., 2003; Olleta et al., 2008). 
Tabla 3. Significación (valor de p) de los efectos estudiados (cruce industrial y tipo de cocinado) sobre la valoración sensorial de la carne de animales procedentes de distintos cruces de Retinta.

Table 3. Signification ( $p$ values) of the studied effects (crossbreeding and cooking method) on the sensory analysis of meat from different Retinta breed crosses.

\begin{tabular}{lccc}
\hline Variable & Cruce & Cocinado & Cruce x Cocinado \\
\hline Aroma característico ternera & 0,156 & 0,723 & 0,667 \\
Aroma sangre & 0,455 & 0,131 & 0,675 \\
Aroma hígado & 0,107 & 0,146 & 0,579 \\
Flavor característico ternera & 0,656 & 0,293 & 0,859 \\
Flavor característico sangre & 0,831 & 0,667 & 0,921 \\
Flavor hígado & 0,668 & 0,071 & 0,635 \\
Flavor grasa & 0,370 & 0,980 & 0,692 \\
Sabor residual & 0,555 & 0,919 & 0,270 \\
Jugosidad inicial & 0,704 & 0,458 & 0,940 \\
Jugosidad continuada & 0,295 & 0,736 & 0,914 \\
Dureza & 0,014 & 0,015 & 0,643 \\
Cohesividad & 0,321 & 0,187 & 0,865 \\
Facilidad tragar & 0,550 & 0,001 & 0,686 \\
Untuosidad & 0,819 & 0,975 & 0,659 \\
\hline
\end{tabular}

Tabla 4. Medias y error estándar para la variable dureza en el análisis sensorial de la carne de animales procedentes de distintos cruces de Retinta.

Table 4. Means and standard error for meat hardness on the sensory analysis of meat from different Retinta breed crosses.

\begin{tabular}{lccccc}
\hline Variable* & RE x RE & LI x RE & PI x RE & AS x RE & e.e. \\
\hline Dureza & $7,12^{\text {ab }}$ & $6,25^{\mathrm{bc}}$ & $7,44^{\mathrm{a}}$ & $5,55^{\mathrm{c}}$ & 0,59 \\
\hline
\end{tabular}

* Escala de 1 a 10, siendo 1 el valor más bajo y 10 el más alto para el atributo. RE = Retinta; LI = Limousine; PI = Pirenaico; AS = Asturiano. e.e.- error estándar. ${ }^{a}, b, c$. - superíndices distintos en la misma fila implican diferencias significativas entre cruces $(p<0,05)$. 
Tabla 5. Medias y error estándar para las variables afectadas por el tipo de cocinado en el análisis sensorial de la carne de animales procedentes de distintos cruces de Retinta.

Table 5. Means and standard error for variables affected by cooking method on the sensory analysis of meat from different Retinta breed crosses.

\begin{tabular}{lcccc}
\hline Variable* $^{*}$ & Cocción & Horno & Plancha & e.e. \\
\hline Dureza & $5,94^{\mathrm{y}}$ & $6,99^{\mathrm{x}}$ & $7,14^{\mathrm{x}}$ & 0,59 \\
Facilidad al tragar & $6,62^{\mathrm{x}}$ & $5,86^{\mathrm{y}}$ & $5,14^{\mathrm{z}}$ & 0,44 \\
\hline
\end{tabular}

* Escala de 1 a 10, siendo 1 el valor más bajo y 10 el más alto para la intensidad del atributo en cuestión. e.e.- error estándar. $x, y, z$.- superíndices distintos en la misma fila implican diferencias significativas entre tipos de cocinado $(p<0,05)$.

\section{Compuestos aromáticos}

En la Tabla 6 se muestran las medias y la significación de los efectos estudiados sobre los compuestos aromáticos detectados. Se identificaron 50 compuestos aromáticos, aunque no todos ellos se encontraron en todas las razas o en todos los tipos de cocinado. Los compuestos se distribuyen en los siguientes grupos: hidrocarburos $(n=27)$, alcoholes $(n=4)$, compuestos azufrados $(n=3)$, cetonas $(n=5)$, ésteres $(n=2)$ y aldehídos $(n=9)$.

El perfil de compuestos volátiles depende de muchos factores, como el tiempo, la temperatura y la técnica de extracción (Lorenzo, 2014).

El cruce tuvo menos influencia que el tipo de cocinado, ya que el primero afectó a 17 de los compuestos volátiles identificados mientras que el cocinado afectó a 41 compuestos. Se encontró una interacción significativa entre efectos para 15 de los compuestos, especialmente hidrocarburos, aunque en aras de una lectura más ágil no se muestra el valor de la interacción en la Tabla 6. Los compuestos afectados por la interacción fueron: 1,1'-oxibis-etano, 2metilpentano, 2-metilheptano, 3-metilheptano, metil-ciclohexano, 1,2,4-trimetil-hexano, tolueno, m-xileno, 1,4-benceno, tiobis-metano, tiourea, acetato de etilo y heptanal.
El cruce afectó al porcentaje de alcoholes, compuestos azufrados y cetonas $(p \leq 0,000)$, pero no al resto de los compuestos $(p>0,05)$. Las diferencias en la proporción de alcoholes se deben sobre todo al etanol. La tiourea sólo se detectó en el cruce REXRE y la concentración de tiobismetano fue menor en el cruce LIxRE que en el resto. La 2-propanona y la 2-butanona son las responsables de las diferencias en la proporción de cetonas y para ambas, el cruce LIXRE presentó valores más bajos y el cruce RExRE valores más altos.

El proceso de calentamiento es fundamental para el desarrollo del perfil de compuestos aromáticos (Mottram, 1998; Domínguez et al., 2014; Khan et al., 2015) y así, la carne cruda mostró, en general, menor concentración y frecuencia de compuestos que la carne cocinada. Tanto en carne cruda como en carne cocinada, los hidrocarburos y las cetonas presentaron mayores concentraciones que el resto de los compuestos. Los hidrocarburos contribuyen muy débilmente al olor de la carne y tienen, además, umbrales de detección muy altos, mientras que los aldehídos y las cetonas, que se producen hacia los $60^{\circ} \mathrm{C}$ por rotura de los lípidos, tienen un umbral de detección bajo (Mottram, 1998). Todos los grupos de compuestos excepto las cetonas se vieron afectados por el tipo de cocinado. 


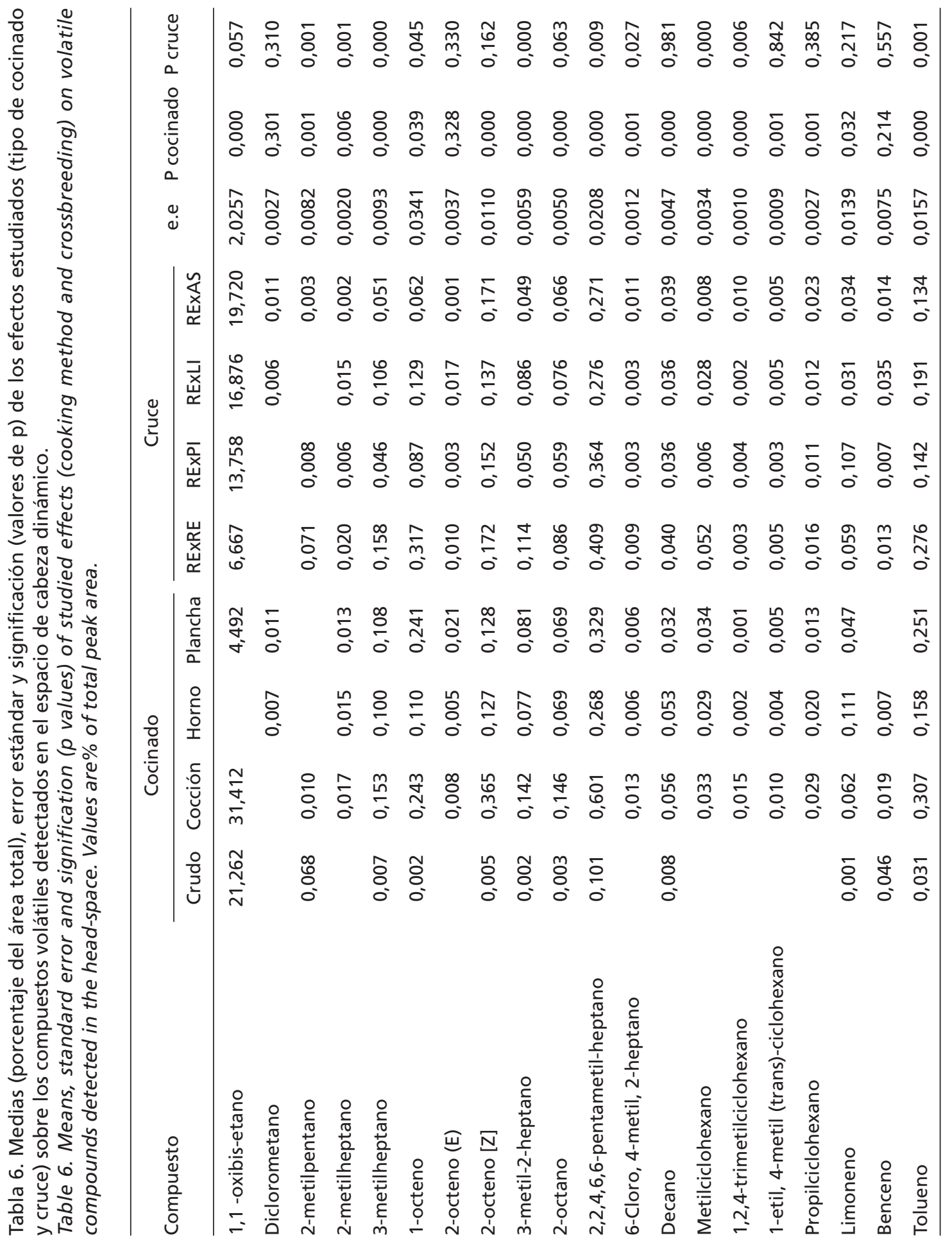




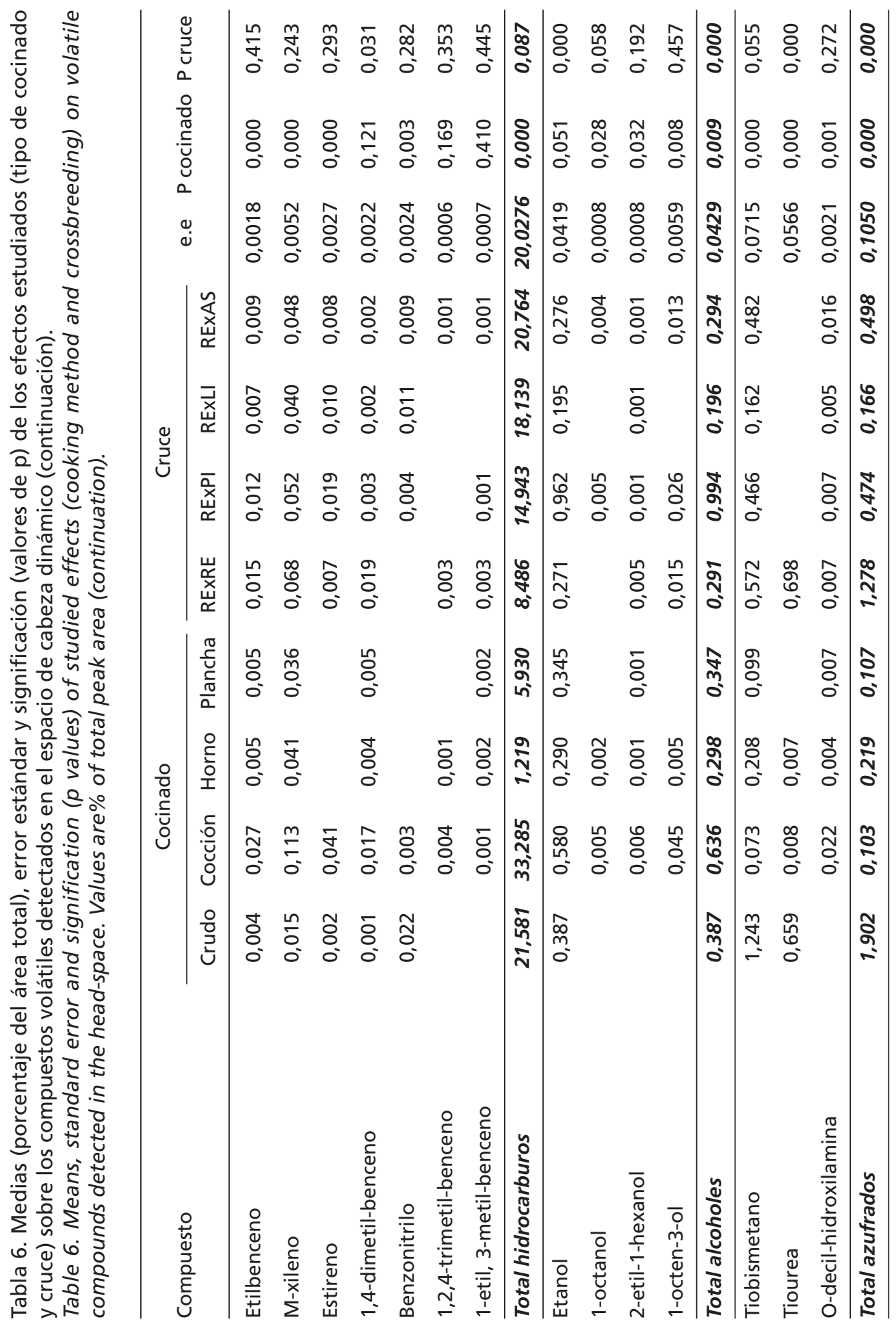




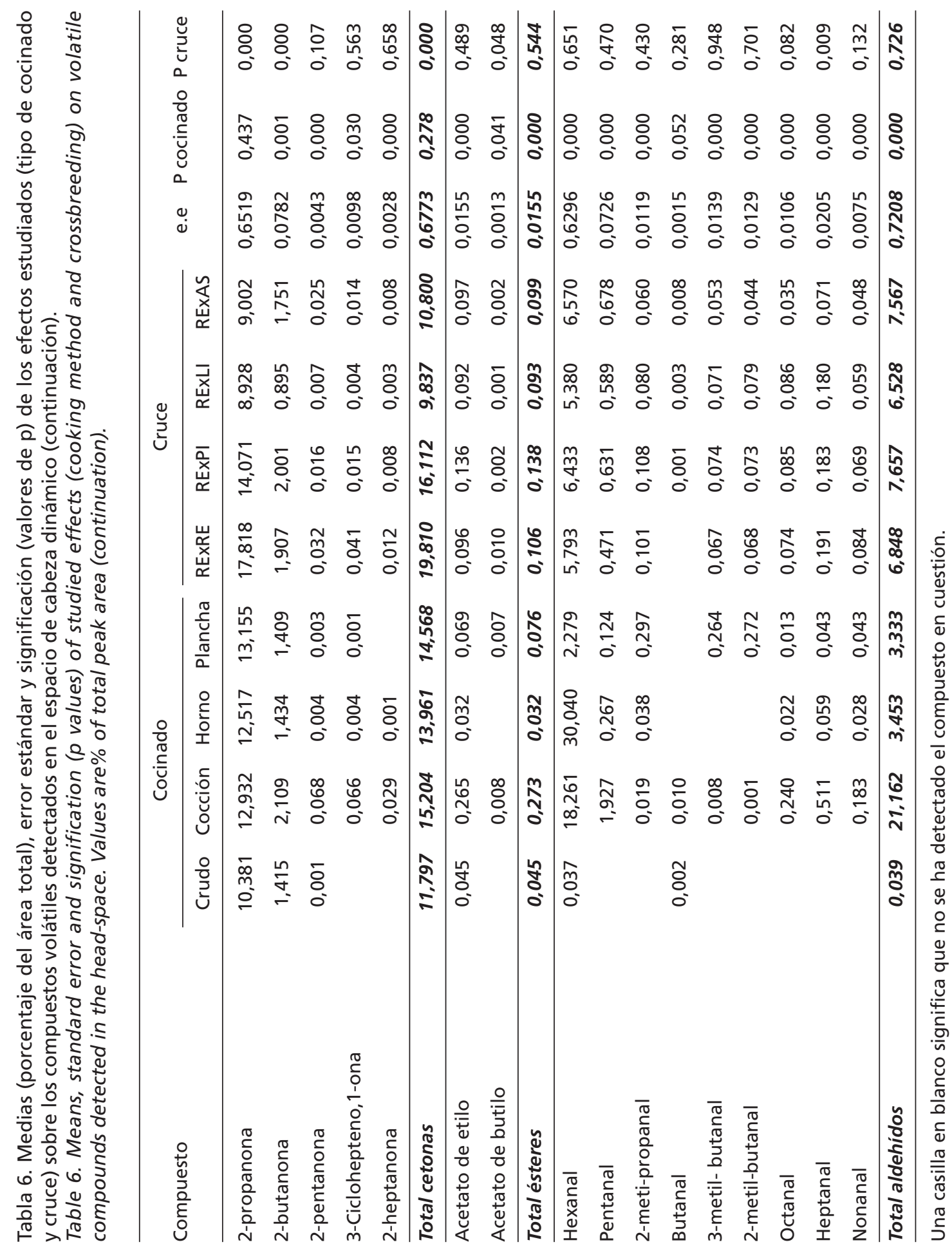


Para todos los grupos de compuestos excepto para los azufrados, las cantidades detectadas fueron mayores cuando el método de cocinado fue la cocción en baño de agua, lo que no se corresponde con las conclusiones generales de la mayoría de los trabajos, según los cuales, al aumentar la temperatura de cocinado aumenta el $\mathrm{n}^{\circ}$ de compuestos volátiles formados (Mottram, 1998; Domínguez et al., 2014). Sin embargo, según Insausti et al. (2005), cuando la temperatura de cocinado no es excesivamente alta $\left(70^{\circ} \mathrm{C}\right.$ en el presente estudio), la mayoría de los compuestos aromáticos se producen por oxidación de los lípidos, lo cual justificaría que en la carne cocinada en baño de agua la concentración de aldehídos sea más elevada que en el resto de los cocinados.

Dentro de los hidrocarburos, los que presentaron mayor concentración fueron el 1, 1'-oxibis-etano, el 2,2,4,6,6-pentametil-heptano y los octenos. El 2,2,4,6,6-pentametilheptano en la carne cruda está relacionado con el flavor a sangre mientras que su presencia en la carne cocinada en plancha se relaciona con el flavor característico a vacuno (Gorraiz et al., 2002). En la carne cocinada al horno, el 1-octeno y el 2-octeno [Z] están relacionados negativamente con el aroma a hígado, por lo que influyen positivamente en la apreciación global. También se encontraron cantidades apreciables de 3-metil-2-hepatno, relacionado positivamente con el flavor característico y de 2-octano, relacionado con el aroma a sangre y el flavor a hígado (Insausti et al., 2002).

El alcohol mayoritario fue el etanol, que aporta un aroma dulce (Revilla et al., 2009). Las dos cetonas que presentaron mayor concentración fueron la 2-propanona y la 2-butanona. Las 2-cetonas son frecuentes en muchos alimentos y dan aromas a mantequilla o queso (Van Ba et al., 2012).

Los ésteres se forman por esterificación de alcoholes y ácidos carboxílicos y tienen um- brales de detección muy bajos (Domínguez et al., 2014). En carne cruda dan olores afrutados pero su presencia en carne cocinada parece deberse a la adición de metanol como estándar interno (Song et al., 2011).

La presencia de aldehídos está relacionada con los procesos de oxidación de los lípidos y entre ellos, el hexanal es el mayoritario debido a que se existen varias rutas que lo originan (Domínguez et al., 2014). Insausti et al. (2005) obtuvieron una correlación negativa del hexanal con el flavor a sangre en carne de vacuno madurada 7 días y cocinada al horno y una relación positiva con el flavor característico en carne madurada 14 días. El octanal y el nonanal, se relacionan con el olor a sopa (Arn y Acree, 1998), mientras que el heptanal da olores pungentes y penetrantes (Domínguez et al., 2014).

En base a los resultados obtenidos, se puede concluir que el cruce no afectó a la valoración sensorial, siendo más importantes el efecto de la maduración y del tipo de cocinado. La terneza, el favor a hígado y el flavor ácido aumentaron con el tiempo de maduración, mientras que la jugosidad disminuyó. El tipo de cocinado afectó a la terneza y a la facilidad al tragar, estando ambas variables inversamente correlacionadas. En el perfil de compuestos aromáticos, el cruce tuvo menos influencia que el tipo de cocinado y se encontró una interacción significativa entre efectos para 15 de los compuestos, especialmente hidrocarburos. El cruce afectó al porcentaje de alcoholes, compuestos azufrados y cetonas. Todos los grupos de compuestos excepto las cetonas se vieron afectados por el tipo de cocinado, siendo los hidrocarburos y las cetonas los compuestos mayoritarios. Para todos los grupos de compuestos excepto para los azufrados, las cantidades detectadas fueron mayores cuando el método de cocinado fue la cocción. 


\section{Agradecimientos}

A Gonzalo Campo, por el trabajo desarrollado. Este trabajo ha sido financiado por el proyecto INIA RTA 2001-106.

\section{Referencias bibliográficas}

Ahumada A (1997). Razas bovinas extranjeras y cruzamientos. En: Vacuno de carne: aspectos claves (Ed. Buxadé C). pp. 135-162. Mundi Prensa Libros SA. Madrid, España.

Albertí P, Sañudo C, Santolaria P, Negueruela I, Olleta JL, Mamaqui E, Campo MM, Álvarez F (1995). Calidad de la carne de terneros de raza Parda Alpina y Pirenaica cebados con pienso rico en gluten feed y mandioca. ITEA-Informacion Tecnica Economica Agraria Vol. Extra 16(2): 630-632.

AMSA (2016). Research guidelines for cookery, sensory evaluation and instrumental tenderness measurements of fresh meat. American Meat Science Association. Chicago, EEUU.

Arn H, Acree TE (1998). Flavornet: A database of aroma compounds based on odor potency in natural products. Developments in Food Science 40 (C): 27.

Campo MM, Sañudo C, Albertí P, Olleta JL, Panea B, Guerrero L (1997). Efecto de la maduración sobre la calidad sensorial de la carne en 7 razas bovinas españolas. ITEA-Informacion Tecnica Economica Agraria Vol. Extra 18: 775-777.

Campo MM, Sañudo C, Panea B, Albertí P, Santolaria $P$ (1999). Breed type and ageing time effects on sensory characteristics of beef strip loin steaks. Meat Science 51: 383-390.

Dickerson GE (1973). Inbreeding and heterosis in animals. Journal of Animal Science 1973(1): 54-77.

Domínguez R, Gómez M, Fonseca S, Lorenzo JM (2014). Effect of different cooking methods on lipid oxidation and formation of volatile compounds in foal meat. Meat Science 97: 223-230.

Font i Furnols M, Guerrero L (2014). Consumer preference, behavior and percepetion about meat and meat products: an overview. Meat Science 98: 361-371.
Gama LT, Bressan MC, Rodrigues EC, Rossato LV, Moreira OC, Alves SP, Bessa RJ (2013). Heterosis for meat quality and fatty acid profiles in crosses among Bos indicus and Bos taurus finished on pasture or grain. Meat Science 93: 98-104.

Gorraiz C, Beriain MJ, Chasco J, Insausti K (2002). Effect of aging time on volatile comounds, odor and flavor of cooked beef from Pireniaca and Friesian bulls and heifers. Journal of Food Science 67: 916-922.

Hughes JM, McPhail NG, Kearney G, Clarke F, Warner RD (2014). Beef longissimus eating quality increases up to 20 weeks of storage and is unrelated to meat colour at carcass grading. Animal Production Science 55: 174-179.

Illescas JL, Ferrer S, Bacho O (2009). Vacuno. Guía práctica. Ed. Mercasa. Madrid, España. 251 pp.

Insausti K, Beriain MJ, Gorraiz C, Purroy A (2002). Volatile compounds of raw beef from 5 local Spanish cattle breeds stored under modified atmosphere. Journal of Food Science 67: 1580-1589.

Insausti K, Goñi V, Petri E, Gorraiz C, Beriain MJ (2005). Effect of weight at slaughter on the volatile compounds of cooked beef from Spanish cattle breeds. Meat Science 70: 83-90.

Kerth CR, Miller RK (2015). Beef flavor: a review from chemistry to consumer. Journal of the Science of Food and Agriculture 95: 2783-2798.

Khan MI, Jo C, Tariq MR (2015). Meat flavor precursors and factors influencing flavor precursors -A systematic review. Meat Science 110: 278-284.

Lorenzo JM (2014). Influence of the type of fiber coating and extraction time on foal dry-cured loin volatile compounds extracted by solidphase microextraction (SPME). Meat Science 96: 179-186.

MacFie HJ, Bratchell N, Greenhoff K, Vallis LV (1989). Designs to balance the effect of order of presentation and first order carry over effects in hall tests. Journal of Sensory Studies 4: 129-148.

Meilgaard M, Civille G, Carr B (1991). Basic statistical methods. En: Sensory Evaluation Techniques, 2nd ed, pp. 237-273. CRC Press, Inc., Boston. 
Mottram DS (1998). Flavour formation in meat and meat products: a review. Food Chemistry 62: 415-424.

Ngapo TM, Riendeau L, Laberge C, Fortin J (2013). Marbling and ageing - Part 2. Consumer perception of sensory quality. Food Research International 51: 985-991.

Olleta JL, Sañudo C, Monsón F, Campo MM, Panea B, Albertí P, Christensen M, Ertbjerg P, Failla S, Gigli S, Hocquette JF, Hughes SI, Williams JL, Nute GR (2008). Sensory evaluation of several European cattle breeds. En: Mediterranean livestock production: uncertainties and opportunities (Eds. Olaizola A, Boutonnet JP, Bernués A) pp. 297-300. CIHEAM / Universidad de Zaragoza / CITA, Zaragoza (España).

Panea B (2002). Influencia de la raza-sistema productivo sobre el tejido conjuntivo y la textura de la carne bovina. Tesis Doctoral. Universidad de Zaragoza. 226 pp.

Panea B, Monsón F, Olleta JL, Martínez-Cerezo S, Pardos JJ, Sañudo C (2003). Estudio textural de la carne de vacuno. II. Análisis sensorial. ITEAInformacion Tecnica Economica Agraria Vol.Extra 24: 31-33.

Panea B, Ripoll G, Sañudo C, Olleta JL, Albertí P (2016). Calidad instrumental de la carne de terneros procedentes del cruce industrial de la raza Retinta. ITEA-Informacion Tecnica Economica Agraria 112(3): 286-300.

Revilla I, Vivar-Quintana AM, Lurueña-Martínez MA, Palacios C, Wilches D, Rovira J, Jaime I (2009). Analisis de compuestos volatiles de carne de lechazo de produccion ecologica y convencional. En: XIII Jornadas sobre Producción Animal, Tomo II (Ed. AIDA), pp. 523-525. INO Reproducciones, S.A. Zaragoza.

Ripoll G, Blanco M, Albertí P, Panea B, Joy M, Casasús I (2014). Effect of two Spanish breeds and diet on beef quality including consumer preferences. Journal of the Science of Food and Agriculture 94: 983-992.

Santolaria P, Sañudo C, Albertí P, Campo MM (1997). Estudio de la calidad organoléptica de la carne de añojo de siete razas españolas mediante una prueba de consumidores. ITEA-Informacion Tecnica Economica Agraria 93A: 89-97.
Sinclair KD, Cuthbertson A, Rutter A, Franklin MF (1998). The effects of age at slaughter, genotype and finishing system on the organoleptic properties and texture of bull beef from suckled calves. Animal Science 66: 329-340.

Song S, Zhang X, Hayat K, Liu P, Jia C, Xia S, Xiao $Z$, Tian H, Niu Y (2011). Formation of the beef flavour precursors and their correlation with chemical parameters during the controlled thermal oxidation of tallow. Food Chemistry 124: 203-209.

Van Ba H, Hwang I, Jeong D, Touseef A (2012). Principle of meat aroma flavors and future prospect. En: Latest Research into Quality Control (Ed. Akya I), pp. 145-176. IntechOpen Limited, London, UK.

Van Den Dool H, Kratz P (1963). A generalisation of the retention index system including linear temperature programmed gas-liquid partition chromatography. Journal of Chromatography A 11: 463-471.

Vieira C, García-Cachán MD, Recio MD, Domínguez M, Sanudo C (2006). Effect of ageing time on beef quality of rustic type and rustic $x$ Charolais crossbreed cattle slaughtered at the same finishing grade. Spanish Journal of Agricultural Research 4: 225-234.

Wood JD, Richardson RI, Nute GR, Fisher AV, Campo MM, Kasapidou E, Sheard PR, Enser M (2004). Effects of fatty acids on meat quality: a review. Meat Science 66: 21-32.

Wood JD, Enser M, Fisher AV, Nute GR, Sheard PR, Richardson RI, Hughes SI, Whittington FM (2008). Fat deposition, fatty acid composition and meat quality: a review. Meat Science 78: 343-358.

Yancey EJ, Dikeman ME, Hachmeister KA, Chambers E, Milliken GA (2005). Flavor characterization of top-blade, top-sirloin, and tenderloin steaks as affected by $\mathrm{pH}$, maturity, and marbling. Journal of Animal Science 83: 2618-2623.

(Aceptado para publicación el 4 de diciembre de 2018) 